\title{
Alpha particles more promising than toxins?
}

\author{
Jacques Barbet • Michel Chérel • Jean-François Chatal
}

Published online: 20 March 2010

(C) Springer-Verlag 2010

The first treatment ever of cancer by radioimmunotherapy (RIT) was performed by William H. Beierwaltes in 1951 and was a success [1]. However, experimentation on this approach really started in 1981 with polyclonal antibodies [2] and radiolabelled monoclonal antibodies were proposed for this purpose by several groups in 1987 and 1988. Research on radioimmunotherapy became more and more active over the years and finally, in 2002 and 2003, radiolabelled monoclonal antibodies, both targeting the CD20 antigen, were approved for marketing for the treatment of follicular non-Hodgkin's lymphoma.

On the other side, immunotoxins were described in 1981 with a pretty advanced description of monoclonal antibodyricin A conjugates [3]. Research in this field was extremely active and many antibodies as well as many different vegetal and bacterial toxins were tested. Toxicity always limited the clinical efficacy with vascular leak syndrome preventing further development in most cases [4]. Shifting from protein toxins to smaller molecules, derived from calicheamicin, auristatin or maytansin, provided better in vivo results [4] and gemtuzumab ozogamicin, an anti-CD33 humanized antibody coupled to calicheamicin obtained market approval in 2000 for the treatment of acute myeloid leukaemia [5].

This Editorial Commentary refers to the article http://dx.doi.org/ 10.1007/s00259-009-1356-x.

J. Barbet $\cdot$ M. Chérel

Nantes-Angers Cancer Research Center, Inserm,

University of Nantes, U892,

Nantes, France

J.-F. Chatal $(\square)$

GIP Arronax, University of Nantes,

Nantes-Saint-Herblain, France

e-mail: chatal@arronax-nantes.fr
Since then, many new radiolabelled antibodies have been proposed, against a variety of antigens, and some have reached the clinic, with good results in haematological diseases and more contrasted outcomes in solid tumours, although evidence of clinical efficacy has been obtained in the treatment of residual disease in metastatic colon cancer [6] or in medullary thyroid carcinoma using a pretargeting technique [7]. The use of alpha particle-emitting radionuclides [8], potentially toxic but also efficient against isolated cells thanks to their high lineic energy transfer, has been proposed for several indications, both in haematological diseases and solid tumours, intracavitary administration being often proposed for the treatment of residual disease. It is expected that alpha particle-emitting radionuclides overwhelm tumour resistance mechanisms by producing clusters of DNA double-stranded breaks (DSB). ${ }^{211} \mathrm{At}$, a cyclotron-produced alpha-emitting radionuclide with relatively long half-life $(7.2 \mathrm{~h})$, is one of a few candidates for targeted alpha-radionuclide therapy [9]. Dose-limiting toxicity is generally haematological, although potential long-term toxicity of alpha-emitting radionuclides is not well known yet.

For the immunotoxin approach, many antibodies conjugated to highly toxic drugs derived from microorganismproduced toxins are in clinical development $[10,11]$. Dose-limiting toxicity appears to be a vaso-occlusive syndrome. Even if the toxins are able to kill chemoresistant tumour cells, gemtuzumab ozogamicin (anti-CD33 conjugated to calicheamicin) is not sufficient to induce persistent complete remission because of resistance mechanisms of leukaemic cells. Since 2008 the clinical use of gemtuzumab ozogamicin is no longer recommended in Europe by the European Medicines Agency (EMEA) because of the combination of poor efficacy and considerable side effects. 
Thus for both immunotoxins and antibody-targeted alpha-emitting radionuclides, a very potent agent, the toxin or the alpha-emitting radionuclide, with little if any capacity to be less toxic to normal tissues than to tumour cells, is proposed to treat cancer diseases. In their paper, entitled "In vitro experimental ${ }^{211}$ At-anti-CD33 antibody therapy of leukaemia cells overcomes cellular resistance seen in vivo against gemtuzumab ozogamicin", Petrich et al. have confronted both approaches in a series of in vitro experiments using an anti-CD33 monoclonal antibody labelled with ${ }^{211}$ At or gemtuzumab ozogamicin. Survival of leukaemic HL-60 and K-562 cells treated with the ${ }^{211}$ Atlabelled antibody, gemtuzumab ozogamicin or control unlabelled antibody were compared. Treatment-induced caspase 3/7 activity, DNA fragmentation and necrosis in HL-60 cells were also compared. As expected, antigen density-dependent specific binding of the antibodies to leukaemic cells was observed and could be specifically blocked by unlabelled anti-CD33. Used at the same antibody concentration, both the ${ }^{211}$ At-labelled anti-CD33 antibody and gemtuzumab ozogamicin were equally potent in induction of apoptosis or necrosis or DNA DSB or in decreasing cell survival.

Interestingly, only 1 in 1,090 antibody molecules actually carried an astatine atom, versus all antibodies carrying at least one toxin molecule in gemtuzumab ozogamicin, with a mean of 2-3 toxins per antibody. If gemtuzumab ozogamicin was used at the same toxin agent concentration (astatine versus toxin), then it was no longer effective against the leukaemic cells.

This observation tells us that antibodies labelled at higher specific activities could be more potent than immunotoxins. This assumes that injected astatine activities may be escalated, but today there is very little information available on the toxicity of astatine-labelled antibodies injected systemically. Most preclinical and clinical studies have been conducted using locoregional administration under conditions where the injected activity was mostly confined to the site of injection.

Nevertheless, results presented in the paper suggest that ${ }^{211}$ At-labelled antibodies, being as potent as and potentially more potent than immunotoxins, are promising, highly cytotoxic radiopharmaceuticals for cancer radioimmunotherapy, which is very good news for nuclear medicine. The authors also state that labelling techniques must be further improved to afford higher yields and specific activities because low specific activity (only 1 labelled antibody molecule in 1,000) limits anti-tumour efficacy when tumour-specific epitopes are sparse. This, and increased availability of ${ }^{211} \mathrm{At}$, are some of the challenges that we are facing today.

\section{References}

1. Srivastava SC. Investigators review potential of antibodies in cancer detection and therapy. J Nucl Med 1987;28:143-7.

2. Goldenberg DM, Gaffar SA, Bennett SJ, Beach JL. Experimental radioimmunotherapy of a xenografted human colonic tumor (GW-39) producing carcinoembryonic antigen. Cancer Res 1981;41(11 Pt 1):4354-60.

3. Blythman HE, Casellas P, Gros O, Gros P, Jansen FK, Paolucci F, et al. Immunotoxins: hybrid molecules of monoclonal antibodies and a toxin subunit specifically kill tumour cells. Nature 1981;290 (5802):145-6.

4. Carter PJ, Senter PD. Antibody-drug conjugates for cancer therapy. Cancer J 2008;14(3):154-69.

5. Stasi R. Gemtuzumab ozogamicin: an anti-CD33 immunoconjugate for the treatment of acute myeloid leukaemia. Expert Opin Biol Ther 2008;8(4):527-40.

6. Liersch T, Meller J, Kulle B, Behr TM, Markus P, Langer C, et al. Phase II trial of carcinoembryonic antigen radioimmunotherapy with 131I-labetuzumab after salvage resection of colorectal metastases in the liver: five-year safety and efficacy results. J Clin Oncol 2005;23(27):6763-70.

7. Chatal JF, Campion L, Kraeber-Bodéré F, Bardet S, Vuillez JP, Charbonnel B, et al. Survival improvement in patients with medullary thyroid carcinoma who undergo pretargeted anticarcinoembryonic-antigen radioimmunotherapy: a collaborative study with the French Endocrine Tumor Group. J Clin Oncol 2006;24(11):1705-11.

8. Kozak RW, Atcher RW, Gansow OA, Friedman AM, Hines JJ, Waldmann TA. Bismuth-212-labeled anti-Tac monoclonal antibody: alpha-particle-emitting radionuclides as modalities for radioimmunotherapy. Proc Natl Acad Sci U S A 1986;83 (2):474-8.

9. Zalutsky MR, Bigner DD. Radioimmunotherapy with alphaparticle emitting radioimmunoconjugates. Acta Oncol 1996;35 (3):373-9.

10. Lewis Phillips GD, Li G, Dugger DL, Crocker LM, Parsons KL, Mai E, et al. Targeting HER2-positive breast cancer with trastuzumab-DM1, an antibody-cytotoxic drug conjugate. Cancer Res 2008;68(22):9280-90.

11. Ikeda H, Hideshima T, Fulciniti M, Lutz RJ, Yasui H, Okawa Y, et al. The monoclonal antibody nBT062 conjugated to cytotoxic Maytansinoids has selective cytotoxicity against CD138-positive multiple myeloma cells in vitro and in vivo. Clin Cancer Res 2009;15(12):4028-37. 